doi:10.1017/S104161021800234X

\section{Compliance and drug-related problems in probable Alzheimer's disease elderly}

Elderly with probable Alzheimer's disease (AD) is more susceptible to Drug Related Problems (DRPs) due to physiological changes (Reeve et al., 2017), cognitive impairment (Mehta et al., 2015) and failure of drug therapy compliance (Smith et al., 2017). Failure of compliance in the elderly with cognitive impairment varies from $10.7 \%$ to $38 \%$, while among the elderly with $\mathrm{AD}$ from $17 \%$ to $100 \%$. (Smith et al., 2017).

In this context, Medication Therapy Management (MTM) is conducted through the identification and resolution of DRPs, evaluation of the therapeutic need, request of examinations of biochemical and physiological parameters for monitoring the effectiveness and safety of drug therapy, based on the underlying disease and the therapeutic experience of the elderly and/or the family/caregiver. Outcomes are also monitored and evaluated.

The MTM was conducted in 55 elderly patients with AD, 39 of whom had compliance problems. After a minimum follow-up of six months and at least six visits/appointments, compliance problems were solved in 30 elderly with AD. The main strategies adopted were medication schedule, unitization of doses per hour of medication intake, substitution for more adequate pharmaceutical form, reminders, and dose adjustments.

The main reasons for non-compliance were the negative therapeutic experience due to adverse drug events and ineffectiveness, mainly due to the mode of use and inadequate pharmaceutical form for the clinical condition, such as dysphagia.

Polypharmacy and the drug therapy complexity have also contributed to compliance problems. Due to lack of caregiver support and/or advanced cognitive impairment of the elderly, only nine elderly with DA remained with compliance problems after the interventions.

The same strategies applied by Oliveira et al. solved half of the DRPs identified in the elderly with cognitive impairment and half of them became compliant (Oliveira et al., 2016).

According to our experience, the MTM providing optimal medication compliance in the elderly with AD, besides solving other DRPs: need (48), effectiveness (17) and safety (21) with a resolution efficiency of $0.83,0.85$, and 0.43 , respectively.

In addition, the integral evaluation of the MTM allowed for adapting the pharmaceutical forms of the drug therapy of one of the elderly with $\mathrm{AD}$ and dysphagia, thus promoting the effectiveness and safety of the drug therapy of depression (sertraline) and hypothyroidism (levotiroxin). The intervention improved the symptoms of major depression and raised the cognitive impairment score. Consequently, the elderly patient was discharged from the governmental protocol of AD (Mastroianni and Forgerini, 2018).

Therefore, it is observed that MTM assessing individual therapeutic needs, prodromal signs and symptoms of $\mathrm{AD}$, and other aging-related comorbidities, has been demonstrated as an effective pharmaceutical service in solving compliance problems and other DRPs.

\section{Conflict of interest}

None.

\section{Funding}

Conselho Nacional para o Desenvolvimento Científico e Tecnológico (CNPq, funding number 459461/ 2014-1 and 131206/2017-6), Fundação de Amparo à Pesquisa do Estado de São Paulo (FAPESP, funding number 2014/00312-5 and 2018/07501-9), PróReitoria de Extensão Universitária da UNESP (PROEX-UNESP), and Coordenação de Aperfeiçoamento de Pessoal de Nível Superior - Brasil (CAPES) - Finance Code 001 all made financial contributions. We, the authors, appreciate all the professionals who collaborate on this pharmaceutical care project.

\section{References}

Mastroianni, P. C. and Forgerini, M. (2018). Drug administration adjustments for elderly patients with dysphagia: a case report. Dementia e Neuropsychologia, 12, 97-100.

Mehta, D. C., Short, J. L., Hilmer, S. N. and Nicolazzo, J. A. (2015). Drug access to the central nervous system in Alzheimer's disease: preclinical and clinical insights. Pharmaceutical Research, 32, 819-839. 
Oliveira, F. M., Lee, S., Carmagnani, O. S. and Mastroianni, P. C. (2016). Indicadores de resultado da implantação da gestão integral da farmacoterapia em um centro de referência do idoso de Araraquara. Experiências Exitosas de Farmacêuticos no SUS, 4, 61-66.

Reeve, E., Trenaman, S. C., Rockwood, K. and Hilmer, S. N. (2017). Pharmacokinetic and pharmacodynamic alterations in older people with dementia. Expert Opinion on Drug Metabolism E Toxicology, 13, 651-668.

Smith, D., et al. (2017). A systematic review of medication nonadherence in persons with dementia or cognitive impairment. PLoS ONE, 12, e0170651.
Patrícia de Carvalho Mastroianni (iD ${ }^{1}$ and Marcela Forgerini ${ }^{2}$

${ }^{1}$ Department of Drugs and Medicines, São Paulo State University, Araraquara, Brazil

${ }^{2}$ Pharmaceutical Sciences Program, São Paulo State University (UNESP), Araraquara, Brazil

Correspondence should be addressed to: Patrícia de Carvalho Mastroianni, School of Pharmaceutical Sciences, São Paulo State University (UNESP), Rodovia Araraquara-Jaú, Km 1, s/n, Campus Ville, Araraquara, SP CEP: 14800-903, Brazil. Email: patriciamastroianni@ yahoo.com.br. Phone: (+55 16) 3301-6977 